\title{
Stability of a Logarithmic Functional Equation in Distributions on a Restricted Domain
}

\author{
Jaeyoung Chung ${ }^{1}$ and Prasanna K. Sahoo ${ }^{2}$ \\ ${ }^{1}$ Department of Mathematics, Kunsan National University, Kunsan 573-701, Republic of Korea \\ ${ }^{2}$ Department of Mathematics, University of Louisville, Louisville, KY 40292, USA
}

Correspondence should be addressed to Jaeyoung Chung; jychung@kunsan.ac.kr

Received 25 May 2013; Accepted 2 August 2013

Academic Editor: Soon-Mo Jung

Copyright ( $(2013$ J. Chung and P. K. Sahoo. This is an open access article distributed under the Creative Commons Attribution License, which permits unrestricted use, distribution, and reproduction in any medium, provided the original work is properly cited.

\begin{abstract}
Let $\mathbb{R}$ be the set of real numbers, $\mathbb{R}^{+}=\{x \in \mathbb{R} \mid x>0\}, \epsilon \in \mathbb{R}_{+}$, and $f, g, h: \mathbb{R}^{+} \rightarrow \mathbb{C}$. As classical and $L^{\infty}$ versions of the Hyers-Ulam stability of the logarithmic type functional equation in a restricted domain, we consider the following inequalities: $|f(x+y)-g(x y)-h((1 / x)+(1 / y))| \leq \epsilon$, and $\|f(x+y)-g(x y)-h((1 / x)+(1 / y))\|_{L^{\infty}\left(\Gamma_{d}\right)} \leq \epsilon$ in the sectors $\Gamma_{d}=\{(x, y): x>$ $0, y>0,(y / x)>d\}$. As consequences of the results, we obtain asymptotic behaviors of the previous inequalities. We also consider its distributional version $\|u \circ S-v \circ \Pi-w \circ R\|_{\Gamma_{d}} \leq \epsilon$, where $u, v, w \in \mathscr{D}^{\prime}\left(\mathbb{R}^{+}\right), S(x, y)=x+y, \Pi(x, y)=x y, R(x, y)=1 / x+1 / y$, $x, y \in \mathbb{R}^{+}$, and the inequality $\|\cdot\|_{\Gamma_{d}} \leq \epsilon$ means that $|\langle\cdot, \varphi\rangle| \leq \epsilon\|\varphi\|_{L^{1}}$ for all test functions $\varphi \in C_{c}^{\infty}\left(\Gamma_{d}\right)$.
\end{abstract}

\section{Introduction}

The Hyers-Ulam stability problem of functional equations was originated in 1940 when Ulam proposed a question concerning the approximate homomorphisms from a group to a metric group (see [1]). A partial answer was given by Hyers et al. $[2,3]$ under the assumption that the target space of the involved mappings is a Banach space. It is possible to prove stability results similar to Hyers for functions that do not have bounded Cauchy difference. In 1950, Aoki [4] first proved such a result for additive functions. Bourgin $[5,6]$ and Aoki [4] studied the Ulam problem from 1949 to 1951. The area rested there for a while until 1978 when Rassias [7] published a generalized version of Hyers' result on linear mappings, where the Cauchy difference was allowed to be unbounded. Rassias' work provided an impetus for the study on the stability of functional equations (see [2, 7-31]).

Let $\mathbb{R}$ be the set of real numbers, $\mathbb{R}_{+}$the set of positive real numbers, and $\mathbb{C}$ the set of complex numbers. The subset, for fixed real number $d>0$,

$$
\Gamma_{d}=\left\{(x, y): x>0, y>0, \frac{y}{x}>d\right\}
$$

of the plane, $\mathbb{R}^{2}$, will be referred to as a sector. A function $f$ : $\mathbb{R}_{+} \rightarrow \mathbb{C}$ is said to be logarithmic if and only if it satisfies the logarithmic functional equation:

$$
f(x y)-f(x)-f(y)=0, \quad \forall x, y \in \mathbb{R}_{+},
$$

for all $x, y \in \mathbb{R}_{+}$. There are several variants of logarithmic functional equations (see [14-16]). It was shown by Heuvers and Kannappan [16] that the logarithmic functional equation is equivalent to the following functional equation:

$$
f(x+y)-f(x y)-f\left(\frac{1}{x}+\frac{1}{y}\right)=0, \quad \forall x, y \in \mathbb{R}_{+} .
$$

They have also studied the following pexiderized version of (3):

$$
f(x+y)-g(x y)-h\left(\frac{1}{x}+\frac{1}{y}\right)=0, \quad \forall x, y \in \mathbb{R}_{+} .
$$

The general solution of the functional equation (4) has the form (see [16])

$$
\begin{gathered}
f(x)=L(x)+c_{1}+c_{2}, \\
g(x)=L(x)+c_{1}, \\
h(x)=L(x)+c_{2},
\end{gathered}
$$


where $L: \mathbb{R}^{+} \rightarrow \mathbb{C}$ is a logarithmic function and $c_{1}, c_{2}$ are arbitrary constants.

In this paper, we study Hyers-Ulam stability of the functional equation (4). In Section 2, we treat the Hyers-Ulam stability of the functional equation (4) in the classical sense and present its asymptotic behavior. In Section 3, we consider the stability of (4) in $L^{\infty}$-sense and its asymptotic behavior. Finally, in Section 4 we present the stability of (4) in Schwartz distributions.

\section{Stability of (4) in Classical Sense and Its Asymptotic Behavior}

In this section, we consider the classical Hyers-Ulam stability of the functional equation (4) on the sector $\Gamma_{d}$ and then study its asymptotic behavior.

The following theorem is a direct consequence of the Hyers' result [3] (see also result of Forti [32]).

Theorem 1. Let $\epsilon$ be a nonnegative real number. Suppose that $f: \mathbb{R}^{+} \rightarrow \mathbb{C}$ satisfies

$$
|f(x y)-f(x)-f(y)| \leq \epsilon
$$

for all $x, y \in \mathbb{R}_{+}$. Then there exists a unique logarithmic function $L: \mathbb{R}^{+} \rightarrow \mathbb{C}$ such that

$$
|f(x)-L(x)| \leq \epsilon, \quad \forall x \in \mathbb{R}_{+} .
$$

Next, we establish the Hyers-Ulam stability of the functional equation (4) on the restricted domain $\Gamma_{d}$.

Theorem 2. Suppose that $\epsilon \geq 0, d>0$, and $f, g$, $h$ satisfy the functional inequality

$$
\left|f(x+y)-g(x y)-h\left(\frac{1}{x}+\frac{1}{y}\right)\right| \leq \epsilon
$$

for all $(x, y) \in \Gamma_{d}$. Then there exists a unique logarithmic function $L: \mathbb{R}^{+} \rightarrow \mathbb{C}$ such that

$$
\begin{gathered}
|f(x)-L(x)-f(1)| \leq 4 \epsilon, \\
|g(x)-L(x)-g(1)| \leq 4 \epsilon, \\
|h(x)-L(x)-h(1)| \leq 4 \epsilon
\end{gathered}
$$

for all $x \in \mathbb{R}_{+}$.

Proof. For given $t, s>0$, choose a real number $u>0$ such that

$$
u \geq \max \left\{\frac{2}{\sqrt{t s^{2}}}, \frac{2}{\sqrt{t s}}, \frac{2}{\sqrt{s}}, \frac{2}{s}, 2 \sqrt{\frac{d}{t s^{2}}}, 2 \sqrt{\frac{d}{t s}}, 2 \sqrt{\frac{d}{s}}, 2 \sqrt{\frac{d}{s^{2}}}\right\},
$$

and let

$$
\begin{array}{cl}
x_{1}=\frac{t s u-\sqrt{t^{2} s^{2} u^{2}-4 t}}{2}, & y_{1}=\frac{t s u+\sqrt{t^{2} s^{2} u^{2}-4 t}}{2}, \\
x_{2}=\frac{t s u-\sqrt{t^{2} s^{2} u^{2}-4 t s}}{2}, & y_{2}=\frac{t s u+\sqrt{t^{2} s^{2} u^{2}-4 t s}}{2}, \\
x_{3}=\frac{s u-\sqrt{s^{2} u^{2}-4 s}}{2}, & y_{3}=\frac{s u+\sqrt{s^{2} u^{2}-4 s}}{2}, \\
x_{4}=\frac{s u-\sqrt{s^{2} u^{2}-4}}{2}, & y_{4}=\frac{s u+\sqrt{s^{2} u^{2}-4}}{2} .
\end{array}
$$

Then it is easy to check that $x_{j}, y_{j}>0, y_{j} / x_{j}>d$ for all $j=1,2,3,4$. Replacing $x, y$ by $x_{j}, y_{j}$ in (8), respectively, for $j=1,2,3,4$ we have

$$
\begin{gathered}
|f(t s u)-g(t)-h(s u)| \leq \epsilon, \\
|f(t s u)-g(t s)-h(u)| \leq \epsilon, \\
|f(s u)-g(s)-h(u)| \leq \epsilon, \\
|f(s u)-g(1)-h(s u)| \leq \epsilon .
\end{gathered}
$$

From (12)-(15), using the triangle inequality we have

$$
|g(t s)-g(t)-g(s)+g(1)| \leq 4 \epsilon
$$

for all $t, s>0$. Similarly, for given $t, s>0$, choose $u>0$ such that

$$
u \geq \max \left\{\frac{4}{t^{2} s}, \frac{4}{t^{2} s^{2}}, \frac{4}{s^{2}}, \frac{4}{s}, \frac{4 d}{t^{2} s}, \frac{4 d}{t^{2} s^{2}}, \frac{4 d}{s^{2}}, \frac{4 d}{s}\right\}
$$

and let

$$
\begin{array}{cl}
x_{1}=\frac{t s u-\sqrt{t^{2} s^{2} u^{2}-4 s u}}{2}, & y_{1}=\frac{t s u+\sqrt{t^{2} s^{2} u^{2}-4 s u}}{2}, \\
x_{2}=\frac{t s u-\sqrt{t^{2} s^{2} u^{2}-4 u}}{2}, & y_{2}=\frac{t s u+\sqrt{t^{2} s^{2} u^{2}-4 u}}{2}, \\
x_{3}=\frac{s u-\sqrt{s^{2} u^{2}-4 u}}{2}, & y_{3}=\frac{s u+\sqrt{s^{2} u^{2}-4 u}}{2}, \\
x_{4}=\frac{s u-\sqrt{s^{2} u^{2}-4 s u}}{2}, & y_{4}=\frac{s u+\sqrt{s^{2} u^{2}-4 s u}}{2} .
\end{array}
$$

Then it is easy to check that $x_{j}, y_{j}>0, y_{j} / x_{j}>d$ for all $j=$ $1,2,3,4$. Next, replacing $x, y$ by $x_{j}, y_{j}$ in (8), respectively, for $j=1,2,3,4$, we have

$$
\begin{gathered}
|f(t s u)-h(t)-g(s u)| \leq \epsilon, \\
|f(t s u)-h(t s)-g(u)| \leq \epsilon, \\
|f(s u)-h(s)-g(u)| \leq \epsilon, \\
|f(s u)-h(1)-g(s u)| \leq \epsilon .
\end{gathered}
$$


From (19), using the triangle inequality, we have

$$
|h(t s)-h(t)-h(s)+h(1)| \leq 4 \epsilon
$$

for all $t, s>0$. Now we prove that

$$
|f(t s)-f(t)-f(s)+f(1)| \leq 4 \epsilon
$$

for all $t, s>0$. For given $t, s>0$, choose $u>0$ such that

$$
u \leq \min \left\{\frac{t^{2} s^{2}}{4}, \frac{t^{2} s}{4}, \frac{s^{2}}{4}, \frac{s}{4}, \frac{t^{2} s^{2}}{4 d}, \frac{t^{2} s}{4 d}, \frac{s^{2}}{4 d}, \frac{s}{4 d}\right\}
$$

and let

$$
\begin{array}{cl}
x_{1}=\frac{t s-\sqrt{t^{2} s^{2}-4 u}}{2}, & y_{1}=\frac{t s+\sqrt{t^{2} s^{2}-4 u}}{2}, \\
x_{2}=\frac{t-\sqrt{t^{2}-4 u / s}}{2}, & y_{2}=\frac{t+\sqrt{t^{2}-4 u / s}}{2}, \\
x_{3}=\frac{s-\sqrt{s^{2}-4 u}}{2}, & y_{3}=\frac{s+\sqrt{s^{2}-4 u}}{2} \\
x_{4}=\frac{1-\sqrt{1-4 u / s}}{2}, & y_{4}=\frac{1+\sqrt{1-4 u / s}}{2} .
\end{array}
$$

Then $x_{j}, y_{j}>0, y_{j} / x_{j}>d$ for all $j=1,2,3,4$. Replacing $x, y$ by $x_{j}, y_{j}$ in (8), respectively, for $j=1,2,3,4$, we have

$$
\begin{aligned}
& \left|f(t s)-g(u)-h\left(\frac{t s}{u}\right)\right| \leq \epsilon, \\
& \left|f(t)-g\left(\frac{u}{s}\right)-h\left(\frac{t s}{u}\right)\right| \leq \epsilon, \\
& \left|f(s)-g(u)-h\left(\frac{s}{u}\right)\right| \leq \epsilon, \\
& \left|f(1)-g\left(\frac{u}{s}\right)-h\left(\frac{s}{u}\right)\right| \leq \epsilon .
\end{aligned}
$$

From (24), using the triangle inequality we get (21).

Now by Theorem 1 , there exist $L_{j}: \mathbb{R}^{+} \rightarrow \mathbb{C}$ for $j=$ $1,2,3$ satisfying the logarithmic functional equation

$$
L_{j}(t s)=L_{j}(t)+L_{j}(s), \quad j=1,2,3,
$$

for which

$$
\begin{aligned}
& \left|f(t)-L_{1}(t)-f(1)\right| \leq 4 \epsilon, \\
& \left|g(t)-L_{2}(t)-g(1)\right| \leq 4 \epsilon, \\
& \left|h(t)-L_{3}(t)-h(1)\right| \leq 4 \epsilon .
\end{aligned}
$$

Now we show that $L_{1}=L_{2}=L_{3}$. Putting $s=u=1$ and $t=u=1$ in (12) separately, we have

$$
\begin{gathered}
|f(t)-g(t)-h(1)| \leq \epsilon \quad \text { for } t \geq \max \{4,4 d\}, \\
|f(s)-h(s)-g(1)| \leq \epsilon \quad \text { for } s \geq \max \{2,2 \sqrt{d}\} .
\end{gathered}
$$

From (26), (27), and (29), using the triangle inequality we have

$$
\begin{aligned}
& \left|L_{1}(t)-L_{2}(t)\right| \\
& \quad \leq 9 \epsilon+|f(1)-g(1)-h(1)| \\
& \quad:=M \quad \text { for } t \geq \max \{4,4 d\} .
\end{aligned}
$$

Let $t>1$. Then we can choose a positive integer $n_{0}$ such that $t^{n} \geq \max \{4,4 d\}$ for all integers $n \geq n_{0}$. In view of (25), and (31) we have

$$
\left|L_{1}(t)-L_{2}(t)\right|=\frac{1}{n}\left|L_{1}\left(t^{n}\right)-L_{2}\left(t^{n}\right)\right| \leq \frac{M}{n}
$$

for all integer $n \geq n_{0}$. Letting $n \rightarrow \infty$ in (32), we have $L_{1}(t)=$ $L_{2}(t)$ for all $t>1$. For $0<t<1$, we have $L_{1}(t)=-L_{1}(1 / t)=$ $-L_{2}(1 / t)=L_{2}(t)$. Thus, we have $L_{1}(t)=L_{2}(t)$ for all $t>0$. Similarly, using (26), (28), and (30) we can show that $L_{1}=L_{3}$. The uniqueness of the logarithmic function $L$ is obvious. This completes the proof of the theorem.

Letting $g=h=f$ in Theorem 2 and using the inequalities (12)-(14) together with the triangle inequality, we obtain

$$
|f(t s)-f(t)-f(s)| \leq 3 \epsilon
$$

for all $t, s>0$. Thus, by Theorem 1 we have the following theorem.

Theorem 3. Let $d>0$. Suppose that $f: \mathbb{R}^{+} \rightarrow \mathbb{C}$ satisfies the functional inequality

$$
\left|f(x+y)-f(x y)-f\left(\frac{1}{x}+\frac{1}{y}\right)\right| \leq \epsilon
$$

for all $(x, y) \in \Gamma_{d}$. Then there exists a unique logarithmic function $L: \mathbb{R}^{+} \rightarrow \mathbb{C}$ such that

$$
|f(x)-L(x)| \leq 3 \epsilon, \quad \forall x \in \mathbb{R}^{+}
$$
(8).

Now we prove the following asymptotic result concerning

Theorem 4. Suppose that $f, g, h: \mathbb{R}^{+} \rightarrow \mathbb{C}$ satisfy the asymptotic condition

$$
\left|f(x+y)-g(x y)-h\left(\frac{1}{x}+\frac{1}{y}\right)\right| \longrightarrow 0
$$

as $(y / x) \rightarrow \infty$. Then there exists a logarithmic function $L:$ $\mathbb{R}^{+} \rightarrow \mathbb{C}$ and $c_{1}, c_{2} \in \mathbb{C}$ such that

$$
\begin{gathered}
f(x)=L(x)+c_{1}+c_{2}, \\
g(x)=L(x)+c_{1}, \\
h(x)=L(x)+c_{2}
\end{gathered}
$$

for all $x>0$. 
Proof. By the condition (36), for any positive integer $n$, there exists $d_{n}>0$ such that

$$
\left|f(x+y)-g(x y)-h\left(\frac{1}{x}+\frac{1}{y}\right)\right| \leq \frac{1}{n}
$$

for all $x, y>0$ with $(y / x)>d_{n}$. By Theorem 1 , there exists a logarithmic function $L_{n}: \mathbb{R}^{+} \rightarrow \mathbb{C}$ such that

$$
\begin{aligned}
& \left|f(x)-L_{n}(x)-f(1)\right| \leq \frac{4}{n} \\
& \left|g(x)-L_{n}(x)-g(1)\right| \leq \frac{4}{n} \\
& \left|h(x)-L_{n}(x)-h(1)\right| \leq \frac{4}{n}
\end{aligned}
$$

for all $x>0$. Replacing $n$ by $m$ in (39) and using the triangle inequality, we have

$$
\left|L_{n}(x)-L_{m}(x)\right| \leq \frac{4}{n}+\frac{4}{m} \leq 8
$$

for all $x>0$. Thus, we obtain

$$
\left|L_{n}(x)-L_{m}(x)\right|=\frac{1}{k}\left|L_{n}\left(x^{k}\right)-L_{m}\left(x^{k}\right)\right| \leq \frac{8}{k}
$$

for all $x>0$ and $k \in \mathbb{N}$. Letting $k \rightarrow \infty$ in (43), we have $L_{n}(x)=L_{m}(x):=L(x)$ for all $x>0$. Finally, letting $n \rightarrow \infty$ in (39), (40), and (41), we have

$$
\begin{aligned}
& f(x)=L(x)+f(1), \\
& g(x)=L(x)+g(1), \\
& h(x)=L(x)+h(1)
\end{aligned}
$$

for all $x>0$. Finally, substituting (44) in (36) we get $f(1)=$ $g(1)+h(1)$. Letting $c_{1}=g(1)$ and $c_{2}=h(1)$ we obtain the asserted result.

\section{Stability of (4) in $L^{\infty}$-Sense and Its Asymptotic Behavior}

In this section, we consider the Hyers-Ulam stability of the functional equation (4) in $L^{\infty}$-sense on the sector $\Gamma_{d}$ and then examine its asymptotic behavior. Consider the functional inequality

$$
\left\|f(x+y)-g(x y)-h\left(\frac{1}{x}+\frac{1}{y}\right)\right\|_{L^{\infty}\left(\Gamma_{d}\right)} \leq \epsilon,
$$

where $\Gamma_{d}=\{(x, y): x>0, y>0,(y / x)>d\}$ and $d>1$ is fixed, where $\|\cdot\|_{L^{\infty}\left(\Gamma_{d}\right)}$ denotes the essential supremum norm of $D(x, y)=f(x+y)-g(x y)-h((1 / x)+(1 / y))$ on the set $\Gamma_{d}$. We employ the function $\delta$ on $\mathbb{R}$ defined by

$$
\delta(x)= \begin{cases}q e^{-\left(1-x^{2}\right)^{-1}}, & \text { if }|x|<1 \\ 0, & \text { if }|x| \geq 1\end{cases}
$$

where

$$
q=\left(\int_{-1}^{1} e^{-\left(1-x^{2}\right)^{-1}} d x\right)^{-1}
$$

It is easy to see that $\delta(x)$ is an infinitely differentiable function with support $\{x:|x| \leq 1\}$. Let $f$ be a locally integrable function and $\delta_{t}(x):=t^{-1} \delta(x / t), t>0$. Then for each $t>0$,

$$
f * \delta_{t}(x)=\int_{-\infty}^{\infty} f(y) \delta_{t}(x-y) d y
$$

is a smooth function of $x \in \mathbb{R}$ and $f * \delta_{t}(x) \rightarrow f(x)$ for almost every $x \in \mathbb{R}$ as $t \rightarrow 0^{+}$.

Now we prove the Hyers-Ulam stability of the functional equation (4) in $L^{\infty}$-sense on the sector $\Gamma_{d}$.

Theorem 5. Let $f, g, h$ be locally integrable functions satisfying (45). Then there exist constants $c_{1}, c_{2}, c_{3}, a \in \mathbb{C}$ such that

$$
\begin{aligned}
& \left\|f(x)-c_{1}-a \ln x\right\|_{L^{\infty}\left(R^{+}\right)} \leq 4 \epsilon, \\
& \left\|g(x)-c_{2}-a \ln x\right\|_{L^{\infty}\left(R^{+}\right)} \leq 4 \epsilon, \\
& \left\|h(x)-c_{3}-a \ln x\right\|_{L^{\infty}\left(R^{+}\right)} \leq 4 \epsilon .
\end{aligned}
$$

Proof. We will use the diffeomorphism

$$
J(x, y)=\left(\ln x y, \ln \frac{x+y}{x y}\right)
$$

Let $u=\ln x y, v=\ln ((x+y) / x y)$ and $y / x=t>1$. Then, we have

$$
\begin{aligned}
u+2 v & =\ln x y+2 \ln \frac{x+y}{x y} \\
& =\ln \left(2+\frac{x}{y}+\frac{y}{x}\right) \\
& =\ln \left(2+t+\frac{1}{t}\right) .
\end{aligned}
$$

Thus, we have $J\left(\Gamma_{d}\right):=U_{d}=\{(u, v): u+2 v>\ln (2+d+1 / d)\}$. Consequently, (45) is converted to

$$
\left\|f\left(e^{u+v}\right)-g\left(e^{u}\right)-h\left(e^{v}\right)\right\|_{L^{\infty}\left(U_{d}\right)} \leq \epsilon
$$

Now, let

$$
F(u)=f\left(e^{u}\right), \quad G(u)=g\left(e^{u}\right), \quad H(u)=h\left(e^{u}\right) .
$$

Then, we have

$$
\|F(u+v)-G(u)-H(v)\|_{L^{\infty}\left(U_{d}\right)} \leq \epsilon .
$$


For each $x, y \in \mathbb{R}$ and $t, s>0$, we have

$$
\begin{aligned}
\iint_{-\infty}^{\infty} & F(u+v) \delta_{t}(x-u) \delta_{s}(y-v) d u d v \\
& =\int_{-\infty}^{\infty} F(u)\left(\int_{-\infty}^{\infty} \delta_{t}(x-u+v) \delta_{s}(y-v) d v\right) d u \\
& =\int_{-\infty}^{\infty} F(u)\left(\int_{-\infty}^{\infty} \delta_{t}(v) \delta_{s}(x+y-u-v) d v\right) d u \\
& =\int_{-\infty}^{\infty} F(u)\left(\delta_{t} * \delta_{s}\right)(x+y-u) d u \\
& =F * \delta_{t} * \delta_{s}(x+y) .
\end{aligned}
$$

We also have

$$
\begin{aligned}
\iint_{-\infty}^{\infty} & G(u) \delta_{t}(x-u) \delta_{s}(y-v) d u d v \\
\quad & \int_{-\infty}^{\infty} G(u) \delta_{t}(x-u)\left(\int_{-\infty}^{\infty} \delta_{s}(y-v) d v\right) d u \\
\quad= & \int_{-\infty}^{\infty} G(u) \delta_{t}(x-u) d u \\
& =G * \delta_{t}(x)
\end{aligned}
$$

Similarly, we have

$$
\iint_{-\infty}^{\infty} H(v) \delta_{t}(x-u) \delta_{s}(y-v) d u d v=H * \delta_{s}(y) .
$$

On the other hand, let $x+2 y>3+\ln (2+d+1 / d)$ and $0<t<1$, $0<s<1$. Then, we have

$$
\begin{aligned}
\operatorname{supp} & \left(\delta_{t}(x-u) \delta_{s}(y-v)\right) \\
\quad= & \{(u, v): x-t \leq u \leq x+t, y-s \leq v \leq y+s\} \subset U_{d} .
\end{aligned}
$$

Let $d^{\prime}=\ln (2+d+1 / d)$. Then it follows from (54) (58) that

$$
\begin{gathered}
\left|F * \delta_{t} * \delta_{s}(x+y)-G * \delta_{t}(x)-H * \delta_{s}(y)\right| \\
=\mid \iint_{-\infty}^{\infty}(F(u+v)-G(u)-H(v)) \\
\times \delta_{t}(x-u) \delta_{s}(y-v) d u d v \mid \\
=\mid \int_{-\infty}^{\infty} \int_{d^{\prime}-2 v}^{\infty}(F(u+v)-G(u)-H(v)) \\
=\iint_{-\infty}^{\infty} \int_{d^{\prime}-2 v}^{\infty}|F(u+v)-G(u)-H(v)| \\
\leq \epsilon \iint_{-\infty}^{\infty}\left|\delta_{t}(x-u) \delta_{s}(y-v)\right| d u d v=\epsilon .
\end{gathered}
$$

Thus, we have the functional inequality

$$
\left|F * \delta_{t} * \delta_{s}(x+y)-G * \delta_{t}(x)-H * \delta_{s}(y)\right| \leq \epsilon
$$

for all $x+2 y>d_{1}:=3+\ln (2+d+1 / d)$ and $0<t<1$, $0<s<1$. From now on, we assume that $0<t<1,0<s<1$. From (60), we have

$$
\begin{aligned}
& \qquad\left|F * \delta_{t} * \delta_{s}(x+y+z)-G * \delta_{t}(x+y)-H * \delta_{s}(z)\right| \leq \epsilon \\
& \text { for } x+y+2 z>d_{1}, \\
& \qquad\left|F * \delta_{t} * \delta_{s}(x+y+z)-G * \delta_{t}(x)-H * \delta_{s}(y+z)\right| \leq \epsilon
\end{aligned}
$$

for $x+2 y+2 z>d_{1}$,

$$
\left|F * \delta_{t} * \delta_{s}(y+z)-G * \delta_{t}(y)-H * \delta_{s}(z)\right| \leq \epsilon
$$

for $y+2 z>d_{1}$,

$$
\left|F * \delta_{t} * \delta_{s}(y+z)-G * \delta_{t}(0)-H * \delta_{s}(y+z)\right| \leq \epsilon
$$

for $2 y+2 z>d_{1}$.

For given $x, y \in \mathbb{R}$, choose $z>(1 / 2)\left(d_{1}+|x|+2|y|\right)$. Then, using the triangle inequality with (61) (64), we have

$$
\left|G * \delta_{t}(x+y)-G * \delta_{t}(x)-G * \delta_{t}(y)+G * \delta_{t}(0)\right| \leq 4 \epsilon
$$

for all $x, y \in \mathbb{R}$. Replacing $(x, t)$ by $(y, s),(y, s)$ by $(x, t)$ in (60) and changing the roles of $G$ and $H$, we have

$$
\left|H * \delta_{t}(x+y)-H * \delta_{t}(x)-H * \delta_{t}(y)+H * \delta_{t}(0)\right| \leq 4 \epsilon
$$

for all $x, y \in \mathbb{R}$. Now we prove that

$$
\left|F * \delta_{t}(x+y)-F * \delta_{t}(x)-F * \delta_{t}(y)+F * \delta_{t}(0)\right| \leq 4 \epsilon
$$

for all $x, y \in \mathbb{R}$. From (60), we have

$$
\begin{gathered}
\left|F * \delta_{t} * \delta_{s}(x+y)-G * \delta_{t}(z)-H * \delta_{s}(x+y-z)\right| \leq \epsilon, \\
\left|F * \delta_{t} * \delta_{s}(x)-G * \delta_{t}(z-y)-H * \delta_{s}(x+y-z)\right| \leq \epsilon, \\
\left|F * \delta_{t} * \delta_{s}(y)-G * \delta_{t}(z)-H * \delta_{s}(y-z)\right| \leq \epsilon, \\
\left|F * \delta_{t} * \delta_{s}(0)-G * \delta_{t}(z-y)-H * \delta_{s}(y-z)\right| \leq \epsilon,
\end{gathered}
$$

for all $x, y, z$ such that $2 x+2 y-z>d_{1}, 2 x+y-z>d_{1}$, $2 y-z>d_{1}$, and $y-z>d_{1}$. For given $x, y \in \mathbb{R}$, choose $z \leq-d_{1}-2|x|-2|y|$. Using the triangle inequality with (68), we have

$$
\begin{aligned}
& \mid F * \delta_{t} * \delta_{s}(x+y)-F * \delta_{t} * \delta_{s}(x)-F * \delta_{t} * \delta_{s}(y) \\
& \quad+F * \delta_{t} * \delta_{s}(0) \mid \leq 4 \epsilon
\end{aligned}
$$

Letting $s \rightarrow 0^{+}$in (69), we get (67). 
Applying Hyers' stability theorem from [3] for (65), (66), and (67), we obtain that for each $0<t<1$ there exist functions $A_{j}(\cdot, t), j=1,2,3$, satisfying

$$
A_{j}(x+y, t)=A_{j}(x, t)+A_{j}(y, t), \quad x, y \in \mathbb{R},
$$

for which

$$
\begin{aligned}
& \left|F * \delta_{t}(x)-A_{1}(x, t)-F * \delta_{t}(0)\right| \leq 4 \epsilon \\
& \left|G * \delta_{t}(x)-A_{2}(x, t)-G * \delta_{t}(0)\right| \leq 4 \epsilon \\
& \left|H * \delta_{t}(x)-A_{3}(x, t)-H * \delta_{t}(0)\right| \leq 4 \epsilon
\end{aligned}
$$

for all $x \in \mathbb{R}$.

Now we prove that $A_{1}=A_{2}=A_{3}$. From (60), using the triangle inequality we have

$$
\left|G * \delta_{t}(x)\right| \leq \epsilon+\left|F * \delta_{t} * \delta_{s}(x+y)\right|+\left|H * \delta_{s}(y)\right|
$$

for all $x+2 y>d_{1}$. Since $F * \delta_{t} * \delta_{s}(x) \rightarrow F * \delta_{s}(x)$ as $t \rightarrow 0^{+}$, in view of (74) it is easy to see that

$$
\widetilde{G}(x):=\limsup _{t \rightarrow 0^{+}} G * \delta_{t}(x)
$$

exists for all $x \in \mathbb{R}$. Similarly, we can show that

$$
\widetilde{H}(x):=\limsup _{s \rightarrow 0^{+}} H * \delta_{s}(x)
$$

exists for all $x \in \mathbb{R}$. Putting $y=0$ in (60) and letting $s \rightarrow 0^{+}$ so that $H * \delta_{s}(0) \rightarrow \widetilde{H}(0)$ we have

$$
\left|F * \delta_{t}(x)-G * \delta_{t}(x)-\widetilde{H}(0)\right| \leq \epsilon
$$

for all $x>d_{1}$. Similarly, we have

$$
\left|F * \delta_{t}(x)-H * \delta_{t}(x)-\widetilde{G}(0)\right| \leq \epsilon
$$

for all $x>\left(d_{1} / 2\right)$. Using (71), (72), (77), and the triangle inequality, we have

$$
\begin{aligned}
\left|A_{1}(x, t)-A_{2}(x, t)\right| & \leq 9 \epsilon+\left|F * \delta_{t}(0)-G * \delta_{t}(0)-\widetilde{H}(0)\right| \\
& :=M(t)
\end{aligned}
$$

for all $x>d_{1}$. From (71) and (80), we have

$$
\begin{aligned}
\left|A_{1}(x, t)-A_{2}(x, t)\right| & =\frac{1}{|k|}\left|A_{1}(k x, t)-A_{2}(k x, t)\right| \\
& \leq \frac{1}{|k|} M(t)
\end{aligned}
$$

for all $x \in \mathbb{R}, x \neq 0$, and all integers $k$ with $k x>d_{1}$. Letting $k \rightarrow \infty$ if $x>0$ and letting $k \rightarrow-\infty$ if $x<0$ in (80), we have $A_{1}(x, t)=A_{2}(x, t)$ for $x \neq 0$, which implies $A_{1}=A_{2}$ since $A_{1}(0, t)=A_{2}(0, t)=0$. Similarly, using (71), (73), and (78) we obtain that $A_{1}=A_{3}$.
Finally, we prove that $A_{1}$ is independent of $t$. Fixing $x \in \mathbb{R}$ and letting $t \rightarrow 0^{+}$so that $G * \delta_{t}(x) \rightarrow \widetilde{G}(x)$ in (60), we have

$$
\left|F * \delta_{s}(x+y)-\widetilde{G}(x)-H * \delta_{s}(y)\right| \leq \epsilon
$$

for all $x+2 y>d_{1}$. From (81), using the same substitutions as in $(61) \sim(64)$ we have

$$
|\widetilde{G}(x+y)-\widetilde{G}(x)-\widetilde{G}(y)+\widetilde{G}(0)| \leq 4 \epsilon .
$$

By Hyers' stability theorem [3], there exists a unique function $A$ satisfying the Cauchy functional equation

$$
A(x+y)-A(x)-A(y)=0
$$

for which

$$
|\widetilde{G}(x)-A(x)-\widetilde{G}(0)| \leq 4 \epsilon .
$$

Now we show that $A_{1}(x, t)=A(x)$ for all $x \in \mathbb{R}$ and $0<t<1$. Putting $y=0$ in (81), we have

$$
\left|F * \delta_{s}(x)-\widetilde{G}(x)-H * \delta_{s}(0)\right| \leq \epsilon
$$

for all $x>d_{1}$. From (71), (84), and (85), using the triangle inequality we have

$$
\left|A_{1}(x, t)-A(x)\right| \leq 9 \epsilon+\left|F * \delta_{t}(0)-H * \delta_{t}(0)-\widetilde{G}(0)\right|
$$

for all $x>d_{1}$. From (86), using the method of proving $A_{1}=$ $A_{2}$ we can show that $A_{1}(x, t)=A(x)$ for all $x \in \mathbb{R}$ and $0<$ $t<1$. Thus, we have $A_{1}=A_{2}=A_{3}:=A$.

Letting $t \rightarrow 0^{+}$in $(72)$ so that $G * \delta_{t}(0) \rightarrow \widetilde{G}(0)$, we have

$$
\|G(x)-A(x)-\widetilde{G}(0)\|_{L^{\infty}} \leq 4 \epsilon .
$$

Similarly, letting $t \rightarrow 0^{+}$in (73) so that $H * \delta_{t}(0) \rightarrow \widetilde{H}(0)$, we have

$$
\|H(x)-A(x)-\widetilde{H}(0)\|_{L^{\infty}} \leq 4 \epsilon .
$$

Now we prove the inequality

$$
\|F(x)-A(x)-\widetilde{F}(0)\|_{L^{\infty}} \leq 4 \epsilon .
$$

For given $x \in \mathbb{R}$, choosing $z$ such that $x+z>d_{1}$ replacing $x$ by $x-z$ and $y$ by $z$ in (81), and using the triangle inequality, we have

$$
\left|F * \delta_{s}(x)\right| \leq \epsilon+\left|\widetilde{G}(x-z)+H * \delta_{s}(z)\right| .
$$

From (90), it is easy to see that

$$
\widetilde{F}(x):=\limsup _{s \rightarrow 0^{+}} F * \delta_{s}(x)
$$

exists for all $x \in \mathbb{R}$. Letting $t \rightarrow 0^{+}$in $(71)$ so that $F * \delta_{t}(0) \rightarrow$ $\widetilde{F}(0)$, we get (89). Replacing $x$ by $\ln x$ in (87), (88), and (89), we have

$$
\begin{aligned}
& \|f(x)-A(\ln x)-\widetilde{F}(0)\|_{L^{\infty}\left(\mathbb{R}^{+}\right)} \leq 4 \epsilon, \\
& \|g(x)-A(\ln x)-\widetilde{G}(0)\|_{L^{\infty}\left(\mathbb{R}^{+}\right)} \leq 4 \epsilon, \\
& \|h(x)-A(\ln x)-\widetilde{H}(0)\|_{L^{\infty}\left(\mathbb{R}^{+}\right)} \leq 4 \epsilon .
\end{aligned}
$$


Finally, we show that the solution $A$ of the Cauchy equation (83) has the form $A(x)=c x$ for some $c \in \mathbb{C}$. Since $\widetilde{G}$ is the supremum limit of a collection of continuous functions $G * \delta_{t}$, $0<t<1, \widetilde{G}$ is a Lebesgue measurable function. Also, as we see in the proof of Hyers-Ulam stability theorem (see [3]), the function $A$ is given by

$$
A(x)=\lim _{n \rightarrow \infty} 2^{-n} \widetilde{G}\left(2^{n} x\right) .
$$

Thus, $A$ is a Lebesgue measurable function since it is the limit of a sequence of Lebesgue measurable functions. It is well known that every Lebesgue measurable solution $A$ of the Cauchy functional equation (83) has the form $A(x)=a x$ for some $a \in \mathbb{C}$. Letting $c_{1}=\widetilde{F}(0), c_{2}=\widetilde{G}(0), c_{3}=\widetilde{H}(0)$ we get the asserted result. (45).

Now we discuss an asymptotic behavior of the inequality

Theorem 6. Let $f, g, h: \mathbb{R}^{+} \rightarrow \mathbb{C}, j=1,2,3$, be locally integrable functions satisfying

$$
\left\|f(x+y)-g(x y)-h\left(\frac{1}{x}+\frac{1}{y}\right)\right\|_{L^{\infty}\left(\Gamma_{d}\right)} \longrightarrow 0
$$

as $d \rightarrow \infty$. Then there exist constants $a, c_{1}, c_{2}, c_{3} \in \mathbb{C}$ such that

$$
\begin{aligned}
& \left\|f(x)-c_{1}-a \ln x\right\|_{L^{\infty}\left(\mathbb{R}^{+}\right)}=0, \\
& \left\|g(x)-c_{2}-a \ln x\right\|_{L^{\infty}\left(\mathbb{R}^{+}\right)}=0, \\
& \left\|h(x)-c_{3}-a \ln x\right\|_{L^{\infty}\left(\mathbb{R}^{+}\right)}=0 .
\end{aligned}
$$

Proof. By the condition (94), for any positive integer $n$ there exists $d_{n}>1$ such that

$$
\left\|f(x+y)-g(x y)-h\left(\frac{1}{x}+\frac{1}{y}\right)\right\|_{L^{\infty}\left(\Gamma_{d}\right)} \leq \frac{1}{n}
$$

for all $x, y>0$ with $(y / x)>d_{n}$. Now by Theorem 5 , there exist constants $a, c_{1}, c_{2}, c_{3} \in \mathbb{C}$ (which are independent of $n$ ) such that

$$
\begin{aligned}
\left\|f(x)-c_{1}-a \ln x\right\|_{L^{\infty}\left(\mathbb{R}^{+}\right)} & \leq \frac{4}{n}, \\
\left\|g(x)-c_{2}-a \ln x\right\|_{L^{\infty}\left(\mathbb{R}^{+}\right)} & \leq \frac{4}{n}, \\
\left\|h(x)-c_{3}-a \ln x\right\|_{L^{\infty}\left(\mathbb{R}^{+}\right)} & \leq \frac{4}{n} .
\end{aligned}
$$

Letting $n \rightarrow \infty$ in (97), we obtain the asserted result.

As a direct consequence of the previous result we have found the solution of functional equation (4) in the $L^{\infty}$-sense.

Corollary 7. Let $f, g, h: \mathbb{R}^{+} \rightarrow \mathbb{C}$ be locally integrable functions satisfying

$$
\left\|f(x+y)-g(x y)-h\left(\frac{1}{x}+\frac{1}{y}\right)\right\|_{L^{\infty}\left(\Gamma_{d}\right)}=0
$$

for all $x, y \in \mathbb{R}_{+}$. Then there exist $a, c_{1}, c_{2}, c_{3} \in \mathbb{C}$ such that

$$
\begin{aligned}
& \left\|f(x)-c_{1}-a \ln x\right\|_{L^{\infty}\left(\mathbb{R}^{+}\right)}=0, \\
& \left\|g(x)-c_{2}-a \ln x\right\|_{L^{\infty}\left(\mathbb{R}^{+}\right)}=0, \\
& \left\|h(x)-c_{3}-a \ln x\right\|_{L^{\infty}\left(\mathbb{R}^{+}\right)}=0 .
\end{aligned}
$$

Finally, we discuss the locally integrable solution $f, g, h$ : $\mathbb{R}^{+} \rightarrow \mathbb{C}$ of the functional equation (c.f. [16])

$$
f(x+y)-g(x y)-h\left(\frac{1}{x}+\frac{1}{y}\right)=0
$$

for all $(x, y) \in \Gamma_{d}$. The following result is a direct consequence of Theorem 2. However, we introduce an alternative proof using Corollary 7. The following method of proof will be useful when we know only regular solution in $L^{\infty}$-sense.

Corollary 8. Every locally integrable solution $f, g, h: \mathbb{R}^{+} \rightarrow$ $\mathbb{C}$ of the functional equation (100) has the form

$$
\begin{gathered}
f(x)=c_{1}+c_{2}+a \ln x, \\
g(x)=c_{1}+a \ln x, \\
h(x)=c_{2}+a \ln x
\end{gathered}
$$

for some constants $a, c_{1}, c_{2} \in \mathbb{C}$.

Proof. It follows from Corollary 7 that (101), (102), and (103) hold in almost everywhere sense; that is, there exists a subset $\Omega \subset \mathbb{R}^{+}$with Lebesgue measure $m\left(\Omega^{c}\right)=0$ such that (101), (102), and (103) hold for all $x \in \Omega$. For given $x>0$, let $p, q$ : $(0, x) \rightarrow \mathbb{R}$ by $p(t)=(1 / t)+(1 /(x-t)), q(t)=t(x-t)$. Since $m\left[\left(p^{-1}(\Omega) \cap q^{-1}(\Omega)\right)^{c}\right]=m\left[p^{-1}\left(\Omega^{c}\right) \cup q^{-1}\left(\Omega^{c}\right)\right]=0$, we can choose $y \in p^{-1}(\Omega) \cap q^{-1}(\Omega)$. Let $z=x-y$. Then $y+z=x$ and $y z,(1 / y)+(1 / z) \in \Omega$. Thus, we can write

$$
\begin{aligned}
f(x) & =g(y z)+h\left(\frac{1}{y}+\frac{1}{z}\right) \\
& =c_{1}+a \ln (y z)+c_{2}+a \ln \left(\frac{1}{y}+\frac{1}{z}\right) \\
& =c_{1}+c_{2}+a \ln (y+z)=c_{1}+c_{2}+a \ln x,
\end{aligned}
$$

which gives (101). For given $x>0$, let $p: \mathbb{R}^{+} \rightarrow \mathbb{R}$ by $p(t)=$ $(1 / t)+(t / x)$. Then, we have $p^{-1}(\Omega) \neq \emptyset$. Choose $y \in p^{-1}(\Omega)$ and let $z=(x / y)$. Then $y z=x,(1 / y)+(1 / z) \in \Omega$. Thus, using (101) we can write

$$
\begin{aligned}
g(x) & =f(y+z)-h\left(\frac{1}{y}+\frac{1}{z}\right) \\
& =c_{1}+c_{2}+a \ln (y+z)-c_{2}-a \ln \left(\frac{1}{y}+\frac{1}{z}\right) \\
& =c_{1}+a \ln (y z)=c_{1}+a \ln x,
\end{aligned}
$$

which gives (102). Finally, (103) follows from (100), (101), and (102). This completes the proof of the corollary. 


\section{Stability of (4) in Schwartz Distributions}

Let $\Omega$ be an open subset of $\mathbb{R}^{n}$. We briefly introduce the space $\mathscr{D}^{\prime}(\Omega)$ of distributions. We denote $\alpha=\left(\alpha_{1}, \ldots, \alpha_{n}\right) \in \mathbb{N}_{0}^{n}$, where $\mathbb{N}_{0}$ is the set of nonnegative integers and $|\alpha|=\alpha_{1}+$ $\cdots+\alpha_{n}, \partial^{\alpha}=\partial_{1}^{\alpha_{1}} \cdots \partial_{n}^{\alpha_{n}}, \partial_{j}=\left(\partial / \partial x_{j}\right), j=1,2, \ldots, n$.

Definition 9. Let $C_{c}^{\infty}(\Omega)$ be the set of all infinitely differentiable functions on $\Omega$ with compact supports. A distribution $u$ is a linear form on $C_{c}^{\infty}(\Omega)$ such that for every compact set $K \subset \Omega$ there exist constants $C>0$ and $k \in \mathbb{N}_{0}$ for which

$$
|\langle u, \varphi\rangle| \leq C \sum_{|\alpha| \leq k} \sup \left|\partial^{\alpha} \varphi\right|
$$

holds for all $\varphi \in C_{c}^{\infty}(\Omega)$ with supports contained in $K$. The set of all distributions is denoted by $\mathscr{D}^{\prime}(\Omega)$.

Let $\Omega_{j}$ be open subsets of $\mathbb{R}^{n_{j}}$ for $j=1,2$, with $n_{1} \geq n_{2}$.

Definition 10. Let $u_{j} \in \mathscr{D}^{\prime}\left(\Omega_{j}\right)$ and let $\lambda: \Omega_{1} \rightarrow \Omega_{2}$ be a smooth function such that for each $x \in \Omega_{1}$ the derivative $\lambda^{\prime}(x)$ is surjective; that is, the Jacobian matrix $\nabla \lambda$ of $\lambda$ has rank $n_{2}$. Then there exists a unique continuous linear map $\lambda^{*}$ : $\mathscr{D}^{\prime}\left(\Omega_{2}\right) \rightarrow \mathscr{D}^{\prime}\left(\Omega_{1}\right)$ such that $\lambda^{*} u=u \circ \lambda$ when $u$ is a continuous function. We call $\lambda^{*} u$ the pullback of $u$ by $\lambda$ and it is usually denoted by $u \circ \lambda$.

If $\lambda$ is a diffeomorphism (a bijection with $\lambda, \lambda^{-1}$ smooth functions) the pullback $u \circ \lambda$ can be written as

$$
\langle u \circ \lambda, \varphi\rangle=\left\langle u,\left(\varphi \circ \lambda^{-1}\right)(x)\left|\nabla \lambda^{-1}(x)\right|\right\rangle .
$$

For more details of distributions we refer the reader to $[29,33]$.

In this section, we consider the Hyers-Ulam stability of the functional equation of (4) in Schwartz distributions, that is, the functional inequality

$$
\|u \circ S-v \circ \Pi-w \circ R\|_{\Gamma_{d}} \leq \epsilon
$$

where $u, v, w \in \mathscr{D}^{\prime}\left(\mathbb{R}^{+}\right), \Pi: \mathbb{R}^{2} \rightarrow \mathbb{R}$, and $R: \mathbb{R}^{2} \rightarrow \mathbb{R}$ are defined by

$$
S(x, y)=x+y, \quad \Pi(x, y)=x y, \quad R(x, y)=\frac{1}{x}+\frac{1}{y}
$$

and the inequality $\|\cdot\|_{\Gamma_{d}} \leq \epsilon$ in (108) means that $|\langle\cdot, \varphi\rangle| \leq$ $\epsilon\|\varphi\|_{L^{1}}$ for all test functions $\varphi \in C_{c}^{\infty}\left(\Gamma_{d}\right)$. For each $t>0, u *$ $\delta_{t}(x)=\left\langle u_{y}, \delta_{t}(x-y)\right\rangle$ is a smooth function of $x \in \mathbb{R}^{n}$ and $u * \delta_{t}(x) \rightarrow u$ as $t \rightarrow 0^{+}$in the sense that

$$
\lim _{t \rightarrow 0^{+}} \int_{-\infty}^{\infty}\left(u * \delta_{t}\right)(x) \varphi(x) d x=\langle u, \varphi\rangle
$$

for all $\varphi \in C_{c}^{\infty}\left(\mathbb{R}^{n}\right)$.
Theorem 11. Let $u, v, w \in \mathscr{D}^{\prime}\left(\mathbb{R}^{+}\right)$satisfy (108). Then there exist constants $a, c_{1}, c_{2}, c_{3} \in \mathbb{C}$ such that

$$
\begin{gathered}
\left\|u-c_{1}-a \ln x\right\| \leq 4 \epsilon, \\
\left\|v-c_{2}-a \ln x\right\| \leq 4 \epsilon, \\
\left\|w-c_{3}-a \ln x\right\| \leq 4 \epsilon .
\end{gathered}
$$

Proof. The idea of the following proof is essentially the same as that of Theorem 5, only with different terminologies. For the reader we give a sketch of proof. Let $u_{d}$ and $J: \Gamma_{d} \rightarrow U_{d}$ be the set and mapping in the proof of Theorem 5 , respectively. Then, $J^{-1}: U_{d} \rightarrow \Gamma_{d}$ is given by

$$
\begin{aligned}
& J^{-1}(x, y) \\
& \quad=\left(\frac{e^{x+y}+\sqrt{e^{2 x+2 y}-4 e^{x}}}{2}, \frac{e^{x+y}-\sqrt{e^{2 x+2 y}-4 e^{x}}}{2}\right) .
\end{aligned}
$$

Taking pullback by $J^{-1}$ in (108), we have

$$
\left\|u \circ E \circ S-v \circ E \circ P_{1}-w \circ E \circ P_{2}\right\|_{U_{d}} \leq \epsilon,
$$

where $E: \mathbb{R} \rightarrow \mathbb{R}, S, P_{1}, P_{2}: \mathbb{R}^{2} \rightarrow \mathbb{R}$ are given by

$$
\begin{array}{lr}
E(x)=e^{x}, \quad S(x, y)=x+y, \\
P_{1}(x, y)=x, \quad P_{2}(x, y)=y .
\end{array}
$$

Thus, instead of (54) we have the inequality

$$
\left\|\tilde{u} \circ S-\tilde{v} \circ P_{1}-\widetilde{w} \circ P_{2}\right\|_{U_{d}} \leq \epsilon,
$$

where $\widetilde{u}=u \circ E, \widetilde{v}=v \circ E$, and $\widetilde{w}=w \circ E$. Using the same approach as in the proof of Theorem 5, we have

$$
\begin{aligned}
& \left\|\tilde{u}-c x-c_{1}\right\| \leq 4 \epsilon, \\
& \left\|\widetilde{v}-c x-c_{2}\right\| \leq 4 \epsilon, \\
& \left\|\widetilde{w}-c x-c_{3}\right\| \leq 4 \epsilon
\end{aligned}
$$

for some $c \in \mathbb{C}$. Taking pullback by $E^{-1}(x)=\ln x$ in (116), we have

$$
\begin{gathered}
\left\|u-a \ln x-c_{1}\right\| \leq 4 \epsilon, \\
\left\|v-a \ln x-c_{2}\right\| \leq 4 \epsilon, \\
\left\|w-a \ln x-c_{3}\right\| \leq 4 \epsilon,
\end{gathered}
$$

for some constants $a, c_{1}, c_{2}, c_{3} \in \mathbb{C}$. This completes the proof of the theorem.

\section{Acknowledgment}

This work was done during the first author's visit to the University of Louisville from Kunsan National University during 2012-13. This work was supported by the Basic Science Research Program through the National Research Foundation of Korea (NRF) funded by the Ministry of Education, Science and Technology (MEST) (no. 2012008507). 


\section{References}

[1] S. M. Ulam, A Collection of Mathematical Problems, Interscience, New York, NY, USA, 1960.

[2] D. H. Hyers, G. Isac, and T. M. Rassias, Stability of Functional Equations in Several Variables, Birkhäuser, Boston, Mass, USA, 1998.

[3] D. H. Hyers, "On the stability of the linear functional equation," Proceedings of the National Academy of Sciences of the United States of America, vol. 27, pp. 222-224, 1941.

[4] T. Aoki, "On the stability of the linear transformation in Banach spaces," Journal of the Mathematical Society of Japan, vol. 2, pp. 64-66, 1950.

[5] D. G. Bourgin, "Classes of transformations and bordering transformations," Bulletin of the American Mathematical Society, vol. 57, pp. 223-237, 1951.

[6] D. G. Bourgin, "Multiplicative transformations," Proceedings of the National Academy of Sciences of the United States of America, vol. 36, pp. 564-570, 1950.

[7] T. M. Rassias, "On the stability of the linear mapping in Banach spaces," Proceedings of the American Mathematical Society, vol. 72, no. 2, pp. 297-300, 1978.

[8] J. A. Baker, "On a functional equation of Aczél and Chung," Aequationes Mathematicae, vol. 46, no. 1-2, pp. 99-111, 1993.

[9] J. A. Baker, “The stability of the cosine equation," Proceedings of the American Mathematical Society, vol. 80, no. 3, pp. 411-416, 1980.

[10] J.-Y. Chung, "Stability of functional equations on restricted domains in a group and their asymptotic behaviors," Computers \& Mathematics with Applications, vol. 60, no. 9, pp. 2653-2665, 2010.

[11] J. Chung, "Stability of approximately quadratic Schwartz distributions," Nonlinear Analysis. Theory, Methods \& Applications A, vol. 67, no. 1, pp. 175-186, 2007.

[12] J. Chung, "A distributional version of functional equations and their stabilities," Nonlinear Analysis. Theory, Methods \& Applications A, vol. 62, no. 6, pp. 1037-1051, 2005.

[13] S. Czerwik, Stability of Functional Equations of Ulam-HyersRassias Type, Hadronic Press, Palm Harbor, Fla, USA, 2003.

[14] Z. Daróczy, "On a functional equation of Hosszú type," Mathematica Pannonica, vol. 10, no. 1, pp. 77-82, 1999.

[15] K. J. Heuvers, "Another logarithmic functional equation," Aequationes Mathematicae, vol. 58, no. 3, pp. 260-264, 1999.

[16] K. J. Heuvers and P. Kannappan, "A third logarithmic functional equation and Pexider generalizations," Aequationes Mathematicae, vol. 70, no. 1-2, pp. 117-121, 2005.

[17] D. H. Hyers and T. M. Rassias, "Approximate homomorphisms," Aequationes Mathematicae, vol. 44, no. 2-3, pp. 125-153, 1992.

[18] S.-M. Jung, Hyers-Ulam-Rassias Stability of Functional Equations in Mathematical Analysis, Hadronic Press, Palm Harbor, Fla, USA, 2001.

[19] S.-M. Jung, "On the Hyers-Ulam stability of the functional equations that have the quadratic property," Journal of Mathematical Analysis and Applications, vol. 222, no. 1, pp. 126-137, 1998.

[20] S.-M. Jung, "Hyers-Ulam-Rassias stability of Jensen's equation and its application," Proceedings of the American Mathematical Society, vol. 126, no. 11, pp. 3137-3143, 1998.

[21] K.-W. Jun and H.-M. Kim, "Stability problem for Jensen-type functional equations of cubic mappings," Acta Mathematica Sinica, vol. 22, no. 6, pp. 1781-1788, 2006.
[22] G. H. Kim, "On the stability of the Pexiderized trigonometric functional equation," Applied Mathematics and Computation, vol. 203, no. 1, pp. 99-105, 2008.

[23] G. H. Kim and Y. W. Lee, "Boundedness of approximate trigonometric functions," Applied Mathematics Letters, vol. 22, no. 4, pp. 439-443, 2009.

[24] C. Park, "Hyers-Ulam-Rassias stability of homomorphisms in quasi-Banach algebras," Bulletin des Sciences Mathématiques, vol. 132, no. 2, pp. 87-96, 2008.

[25] J. M. Rassias and M. J. Rassias, "On the Ulam stability of Jensen and Jensen type mappings on restricted domains," Journal of Mathematical Analysis and Applications, vol. 281, no. 2, pp. 516524, 2003.

[26] J. M. Rassias, "On the Ulam stability of mixed type mappings on restricted domains," Journal of Mathematical Analysis and Applications, vol. 276, no. 2, pp. 747-762, 2002.

[27] J. M. Rassias, "On approximation of approximately linear mappings by linear mappings," Journal of Functional Analysis, vol. 46, no. 1, pp. 126-130, 1982.

[28] T. M. Rassias, "On the stability of functional equations in Banach spaces," Journal of Mathematical Analysis and Applications, vol. 251, no. 1, pp. 264-284, 2000.

[29] L. Schwartz, Théorie des Distributions, Hermann, Paris, France, 1966.

[30] F. Skof, "On the approximation of locally $\delta$-additive mappings," Atti della Accademia delle Scienze di Torino, vol. 117, no. 4-6, pp. 377-389, 1983.

[31] F. Skof, "Proprieta' locali e approssimazione di operatori," Rendiconti del Seminario Matematico e Fisico di Milano, vol. 53, no. 1, pp. 113-129, 1983.

[32] G. L. Forti, "The stability of homomorphisms and amenability, with applications to functional equations," Abhandlungen aus dem Mathematischen Seminar der Universität Hamburg, vol. 57, pp. 215-226, 1987.

[33] L. Hörmander, The Analysis of Linear Partial Differential Operator I, Springer, Berlin, Germany, 1983. 


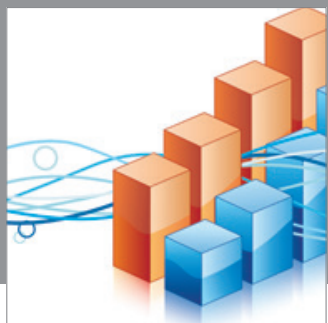

Advances in

Operations Research

mansans

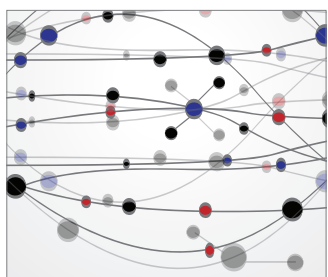

The Scientific World Journal
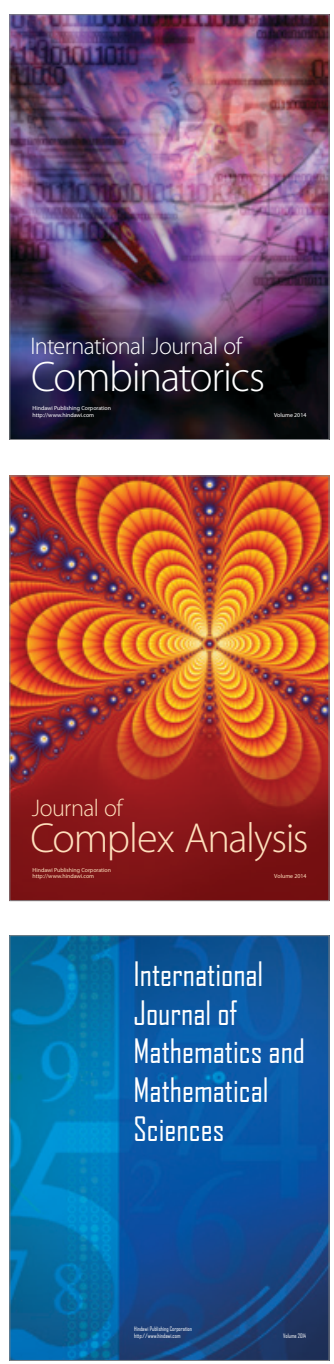
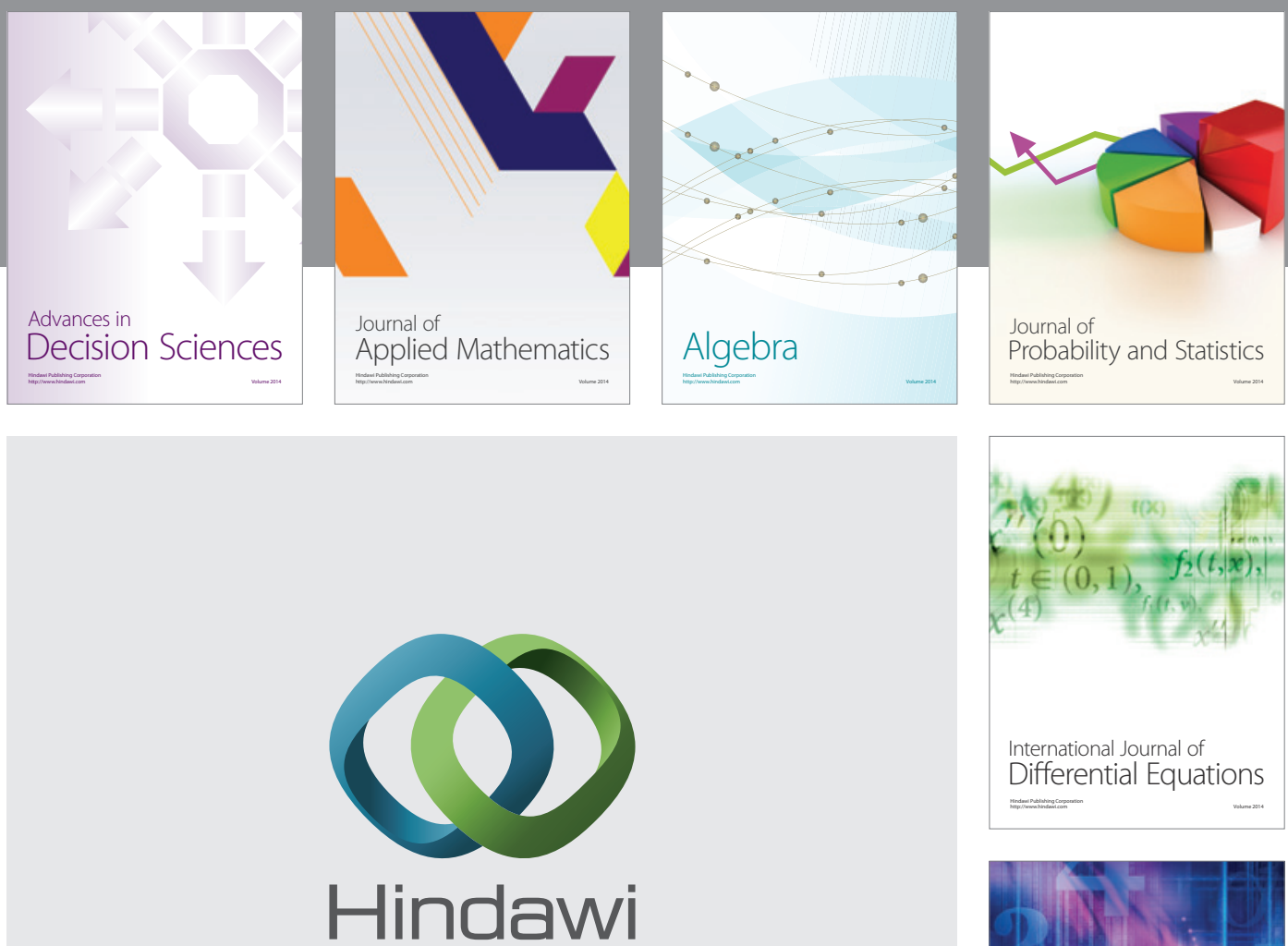

Submit your manuscripts at http://www.hindawi.com
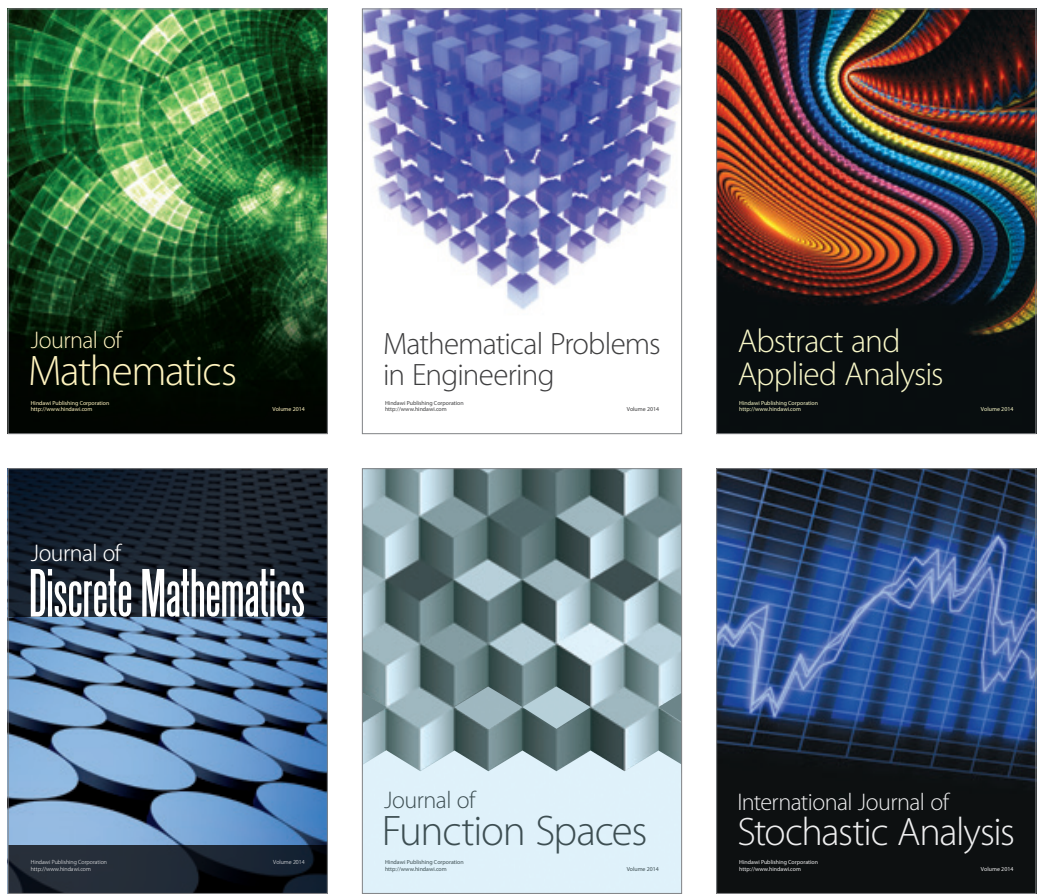

Journal of

Function Spaces

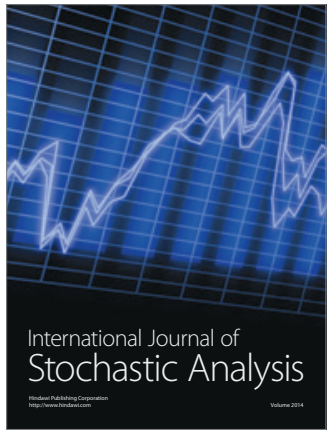

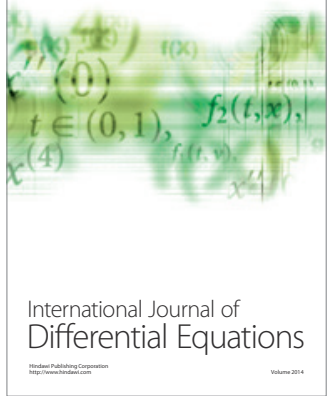
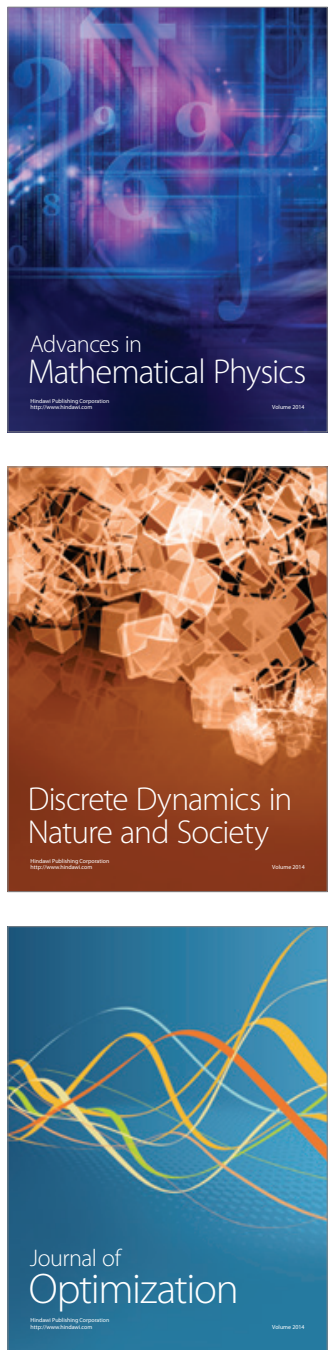